\title{
Cistinuria: diagnóstico y aproximación terapéutica
}

\section{Cystinuria: diagnosis and therapeutic approach}

\section{C.M. Cabrera-Morales}

\section{RESUMEN}

La cistinuria es una aminoaciduria debida a un transporte defectuoso de cistina y de aminoácidos dibásicos (arginina, ornitina, y lisina) en la membrana apical del epitelio intestinal y túbulo proximal renal. El resultado es una ausencia de reabsorción de cistina en el túbulo proximal renal produciendo un exceso de cistina en orina y con la consiguiente formación de cálculos renales. Los cálculos de cistina son muy difíciles de eliminar por litotricia a diferencia del resto de cálculos. Por lo tanto, debería de llevarse a cabo una terapia no invasiva para prevenir la recidiva en la formación de cálculos. Esta terapia estaría basada en una alta ingesta de líquidos, alcalinización de la orina, y empleo de agentes quelantes. A la hora de preservar la función renal es necesaria la combinación de estas tres medidas terapéuticas tanto para disminuir la recurrencia como la morbilidad de la enfermedad.

Palabras clave. Cistinuria. Urolitiasis. Terapia. Agentes quelantes.

\begin{abstract}
Cystinuria is an aminoaciduria due to the impairment of transport of cystine and dibasic amino acids (arginine, ornithine, and lysine) in the apical membrane of the intestinal epithelium and proximal renal tubule. The result is an absence of cystine reabsorption in the renal tubule producing an excess of cystine in urine and stone formation. Unlike the other stones, cystine stones are very difficult to eliminate with lithotripsy. Noninvasive therapy should therefore be used to prevent relapse in stone formation. This therapy is based on the use of high fluid intake, urine alkalinization, and chelating agents. In order to preserve renal function, a combination of these three therapeutic measures is necessary to produce a low recurrence and morbidity of the disease.
\end{abstract}

Key words. Cystinuria. Urolithiasis. Therapy. Chelating agents.
Servicio de Análisis Clínicos. Hospital General Universitario. Ciudad Real.

Recepción: 2 de junio de 2011

Aceptación provisional: 21 de junio de 2011

Aceptación definitiva: 28 de junio de 2011

\section{Correspondencia:}

\section{C.M. Cabrera}

Hospital General Universitario de Ciudad Real Servicio de Análisis Clínicos

C/ Obispo Rafael Torija, s/n

13005 Ciudad Real

E-mail: carmenmcabrera@gmail.com 


\section{INTRODUCCIÓN}

La cistinuria se engloba dentro de las alteraciones congénitas del transporte de aminoácidos, resultando en un aumento de la excreción urinaria de cistina y de aminoácidos dibásicos (ornitina, lisina, y arginina) (COLA) (OMIN 220100). Su incidencia varía según las series estudiadas con una prevalencia promedio de 1/7.000 nacimien$\operatorname{tos}^{1}$. A pesar de verse incrementados los niveles urinarios de varios aminoácidos, únicamente el aumento de los niveles de cistina excretados produce síntomas. Siendo la formación de cálculos urinarios de cistina la única manifestación clínica conocida, y que por ende da nombre a la enfermedad ${ }^{2}$.

El defecto congénito se encuentra en un transportador localizado en el borde en cepillo apical de las células del túbulo recto proximal renales (segmento S3) y en los enterocitos intestinales, el cual pertenece a una familia de transportadores conocidos como HAT Heteromeric Aminoacid Transporter (transportador de aminoácidos heterodimérico $)^{3,4}$. Dentro de esta familia de transportadores, el que está implicado en concreto en la cistinuria se conoce como $\mathrm{b}^{0,+}$, el cual está formado por dos proteínas: una cadena pesada o rBAT codificada por el gen SLC3A1 (SLC de solute carrier) (cromosoma 2, locus 2p16.3-21), y una cadena ligera denominada $\mathrm{b}^{0,+} \mathrm{AT}$ codificada por el gen SLC7A9 (cromosoma 19, locus 19q12$13)^{5}$. La cadena pesada rBAT es una glicoproteína transmembrana de un solo paso tipo II con 685 aminoácidos y un gran dominio extracelular. La cadena ligera $\mathrm{b}^{0,+} \mathrm{AT}$ es una proteína no-glicosilada transmembrana multipaso tipo I de 487 aminoácidos y con 12 dominios transmembrana ${ }^{6}$. Ambas proteínas se unen entre sí por un puente disulfuro en el cual interviene una Cys localizada en el gran dominio extracelular de la cadena pesada y otra Cys localizada en el asa extracelular entre las hélices 3 y 4 de la cadena ligera. En el heterodímero es la cadena ligera la que interviene realmente en el transporte de aminoácidos ${ }^{7}$. El sistema tiene baja especificidad y alta afinidad (Km pequeña) comparado con otros sistemas de transporte de aminoácidos, intercambia cistina y aminoácidos dibásicos (Lys, Arg, Orn) extracelulares por aminoácidos neutros intracelulares con una estequiometría 1:1 (Fig. 1) ${ }^{8}$.

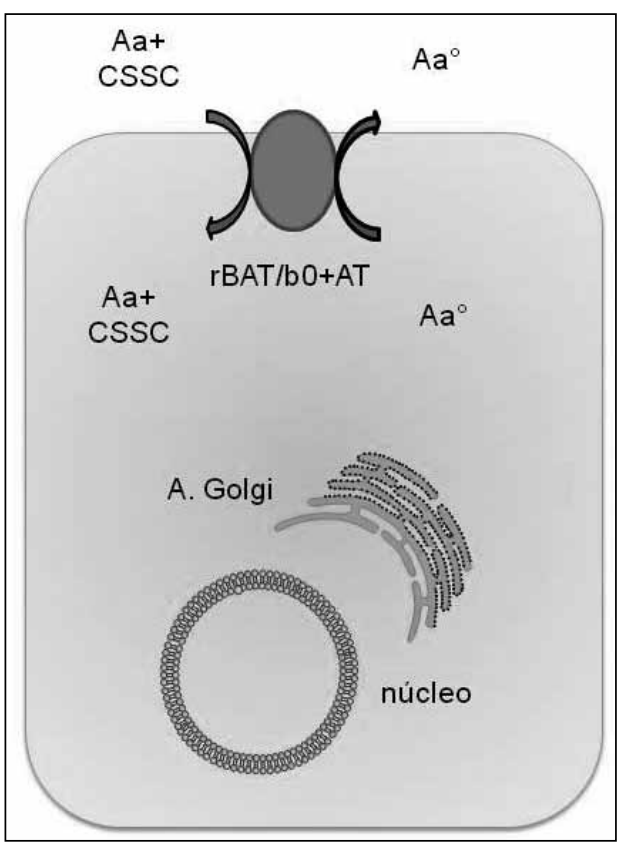

Figura 1. Transporte de aminoácidos dibásicos y de cistina a través del polo apical de una célula del túbulo proximal renal. Aa+, aminoácido dibásico; $\mathrm{Aa}^{\circ}$, aminoácido neutro; $\mathrm{CSSC}$, cistina.

La cistina que se forma a partir de la oxidación de dos moléculas de cisteína (Cys) (Fig. 2A) es un compuesto altamente insoluble, que al no poder reabsorberse a nivel renal por defecto en el transportador específico $\mathrm{b}^{0,+}$ precipita a $\mathrm{pH}$ ácido en los conductos renales dando lugar a la aparición de cálculos de cistina. En condiciones normales en el riñón se reabsorben más del 95\% de los aminoácidos filtrados, sin embargo en los pacientes con cistinuria casi el $100 \%$ de la cistina filtrada se excreta en la orina. Los cálculos de cistina representan únicamente el 1-2\% del total de cálculos, pero en la edad pediátrica representan hasta el $6-8 \%$ del total ${ }^{9}$. Son cálculos de aspecto coraliforme, duros, radio-opacos y con una tasa de recidiva muy alta de ahí la gran importancia que tiene la profilaxis 
en la prevención de la aparición de nuevos cálculos. Lo que exige plantear una estrategia de tratamiento específica en estos pacientes, diferente de la empleada en otras litiasis para prevenir en la medida de lo posible la futura aparición de cálculos y sus complicaciones. La enfermedad tiene la misma incidencia en ambos sexos, pero en los hombres la tasa de morbilidad es mayor debido precisamente a la anatomía del aparato urinario masculino que favorece una mayor probabilidad de obstrucción de la uretra ${ }^{10}$. Los cálculos de cistina además de producir obstrucción, resultan en importantes infecciones urinarias con piuria, presencia de hematuria micro o macroscópica, cólico nefrítico (con o sin expulsión de cálculo), y dolor lumbar ${ }^{7}$. En casos aislados la cistinuria puede progresar hacia una insuficiencia renal aguda o crónica siendo necesaria la diálisis ${ }^{11}$, habiéndose encontrado hasta un $17 \%$ de pacientes cistinúricos con disminución de la tasa de filtrado glomerular. Igualmente se ha observado que los pacientes con cistinuria comparados con los formadores de cálculos de oxalato de calcio tienen una probabilidad 5 veces mayor de sufrir pérdida de la función renal ${ }^{11}$. La frecuencia en la formación de cálculos varía entre unas pocas veces a lo largo de la vida en las formas más leves hasta varias por año en las formas más graves con debut en la primera década de la vida $^{12}$.

A)
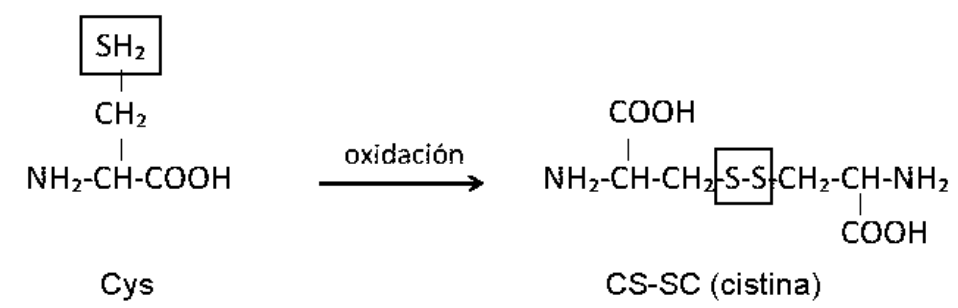

CS-SC (cistina)

B)

\section{$\mathrm{CS}-\mathrm{SC}+$ PENICILAMINA $\longrightarrow \mathrm{COOH}-\mathrm{CHNH}_{2}-\mathrm{CH}_{2}-\mathrm{S}-\mathrm{S}-\mathrm{C}-\left(\mathrm{CH}_{3}\right)_{2}-\mathrm{CHNH}_{2}-\mathrm{COOH}$}

\section{COMPLEJO CISTEINA-PENICILAMINA}

Figura 2. A) Formación de una molécula de cistina (CSSC) a partir de la oxidación de dos moléculas de cisteína (Cys). B) Acción quelante de la D-penicilamina sobre la cistina, con formación de un complejo cistina-penicilamina soluble.

Antes de conocer las bases genéticas de la enfermedad se estableció una primera clasificación basada en la capacidad fenotípica de formar cálculos ${ }^{7}$ :

A. Tipo I: forma recesiva completa siendo los homocigotos formadores de cálculos (>420 mM cistina/orina 24 horas), y los heterocigotos portadores no formadores ( $<420 \mathrm{mM}$ cistina/ orina 24 horas).
B. Tipo no-I: forma recesiva "incompleta" o "co-dominante", presentando tanto los homocigotos como los portadores heterocigotos valores de cistina en grado variable formadores de cálculos ( $>420 \mathrm{mM}$ cistina/orina 24 horas).

Con posterioridad, el Consorcio Internacional de Cistinuria (ICC) gracias al conocimiento de los genes implicados propuso 
una nueva clasificación que correlacionaba el fenotipo-genotipo, siendo la empleada mayormente en la actualidad ${ }^{5,13}$

1. Cistinuria Tipo A: causada por mutaciones homocigotas en ambos alelos del gen SLC3A1 que codifica la cadena pesada. Los heterocigotos muestran un patrón de excreción urinaria de aminoácidos dentro de la normalidad. Es el tipo más frecuente representando hasta el $60 \%$ del total de casos, siendo además el fenotipo más severo, con un mayor riesgo litogénico y con aparición a edad más temprana ${ }^{2}$.

2. Cistinuria Tipo B: heterocigota incompleta causada por una mutación en el gen SLC7A9 que codifica la cadena ligera. De forma que los individuos homocigotos, y los portadores heterocigotos de un alelo mutado presentan fenotípicamente la formación de cálculos pero con una menor severidad que los Tipo A (es una forma más moderada). Solamente en un $14 \%$ de los heterocigotos tipo B el patrón de aminoaciduria es normal ${ }^{13}$.

3. Cistinuria Tipo AB: forma heterocigota compuesta producida por sendas mutaciones en ambos genes SLC3A1 y SLC7A9. Es el tipo más infrecuente representando únicamente el $2 \%$ del total de casos de cistinuria descritos y con un riesgo litogénico leve ${ }^{14}$.

Desde el punto de vista genético es una enfermedad con una gran heterogeneidad, como lo demuestra el hecho de la gran variedad de mutaciones encontradas. Hasta la fecha se han contabilizado un total de 133 mutaciones diferentes en la cadena pesada y 95 en la cadena ligera ${ }^{15}$. Siendo además su incidencia muy variable dependiendo de las distintas áreas geográficas y razas estudiadas, desde los $1 / 2.500$ casos en los judíos libios hasta los 1/100.000 de la población sueca ${ }^{16}$.

\section{DIAGNÓSTICO BIOQUÍMICO}

Niveles en orina superiores a $113 \mathrm{mM}$ de cistina/mM de creatinina o $>420 \mathrm{mM}$ / orina de 24 horas se han descrito como generadoras de litiasis ${ }^{17}$.

En la detección de la presencia de cistina en la orina de pacientes con sospecha de presentar la enfermedad se han empleado diversos métodos. El más antiguo pero que todavía tiene vigencia es el Test cualitativo de Brand (Test de ciano-nitroprusiato sódico). En este test cuando el cianuro de sodio se mezcla con orina alcalinizada, la cistina es reducida a cisteína que en presencia de nitroprusiato de sodio da un color magenta característico. Esta reacción es positiva en pacientes con niveles de cistina $>315 \mathrm{mM} /$ $\mathrm{mM}$ de creatinina, por lo que sirve para detectar únicamente pacientes litogénicos del tipo A homocigotos pero no detecta ni los tipos B heterocigotos ni tampoco los $\mathrm{AB}$. Es un test con una sensibilidad del $72 \%$ y una especificidad del $95 \%$; que sin embargo se pueden cometer falsos positivos como en el caso de pacientes con presencia de homocistinuria y con cetonuria ${ }^{1}$. Todavía el Test de Brand se sigue usando como método de screening neonatal en comunidades autónomas como Murcia y Valencia ${ }^{9,19}$.

El procedimiento diagnóstico más sencillo es la visualización directa en el sedimento urinario de los cristales hexagonales planos y transparentes típicos de cistina, aunque solamente se detectan en la orina de un bajo número de pacientes, entre el $19-26 \%$ de los pacientes homocigotos ${ }^{7}$. Estos cristales son más fácilmente observables en la primera orina de la mañana, ya que ésta es más ácida y está más concentrada. Sin embargo en pacientes con cistinuria donde no se hallan visualizado los cristales en el sedimento, si la acidificamos con ácido acético conseguiremos que precipiten haciéndose visibles. También tenemos que considerar que la aparición en la orina de cristales hexagonales de cistina no es patognomónica de la cistinuria. Ya que igualmente se pueden encontrar estos cristales en la orina de pacientes con cistinosis (OMIN 219800): una enfermedad de almacenamiento lisosomal en la cual se acumulan cristales hexagonales de cistina en los lisosomas de varios tejidos (riñón, corazón, y tejido muscular entre otros) ${ }^{20}$. La cistinosis 
es una enfermedad con una incidencia muy baja (1/100.000-200.000) en comparación con la cistinuria, que cursa además con Síndrome de Fanconi (aminoaciduria con cistinuria, bicarbonaturia, fosfaturia, acidosis tubular, etc.) causado por nefropatía por acúmulo de cristales de cistina en las células tubulares. Sin embargo, no da lugar a la aparición de cálculos de cistina por lo que es muy poco probable confundirla con la cistinuria, además de por la aparición de otros síntomas asociados.

En la actualidad los métodos diagnósticos que ofrecen una mayor especificidad y sensibilidad en el análisis de la composición química de los cálculos de cistina son el método químico semi-cuantitativo y los métodos físicos, ofreciendo ambos tipos resultados similares. Mediante el método químico semi-cuantitativo como el de DiaSYS (DyaSys GmbH, Diagnostic Systems $\mathrm{GmbH})$ se puede analizar de forma muy simple la composición química de cualquier tipo de cálculo urinario incluyendo los de cistina. En concreto para determinar la composición de un cálculo de cistina: la cistina se reduce a cisteína con un agente reductor (sulfito de sodio) y en medio alcalino (solución de amonio) la cisteína resultante da una coloración roja característica en presencia del nitroprusiato de sodio. El DiaSys tiene un intervalo de detección que va desde 10 a $100 \mathrm{mg}$ de cistina por cálculo. Dentro de los métodos físicos de examen de cálculos, la espectrometría infrarroja y de rayos $\mathrm{X}$ ofrecen igualmente muy buenos resultados. Sin embargo el análisis químico semi-cuantitativo como el de DiaSys es un método muy económico que está disponible en cualquier laboratorio de análisis clínicos ofreciendo una alternativa muy rápida y simple para el diagnóstico de los cálculos de cistina.

Cuando los cálculos son exclusivamente de cistina tienen un color amarillo "miel", con un brillo perlado y son radioopacos debido a la densidad de las moléculas de sulfuro ${ }^{10}$. Sin embargo, un $50 \%$ de los cálculos encontrados en los pacientes cistinúricos no son puros y se encuentran asociados con otras sales como son el oxa- lato de calcio, la estruvita (fosfato-amónico-magnésico), y con el ácido úrico ${ }^{7}$.

A la hora de evaluar los niveles de cistina urinaria durante la repuesta al tratamiento la cromatografía líquida de alta resolución (HPLC) con derivatización de pre-columna ofrece un buen método, y que además se puede usar para el diagnóstico si existe sospecha ${ }^{21}$. Con la cromatografía de intercambio iónico niveles de cistina menores de $30 \mu \mathrm{mol} / \mathrm{mmol}$ de creatinina estarían dentro de la normalidad, y los mayores de $100 \mu \mathrm{mol}$ de cistina/mmol de creatinina serían indicativos de cistinuria.

\section{PERSPECTIVAS TERAPÉUTICAS}

Ante un paciente con diagnóstico de cistinuria, hay que plantear una estrategia en parte común a la empleada en otras litiasis renales. Pero dada las características propias que presentan los cálculos de cistina es preferible utilizar como primera línea de elección en el tratamiento de los mismos una terapia conservadora frente a la litofragmentación. Los cálculos de cistina son de gran dureza, por lo que la litotricia extracorpórea por ondas de choque (LEOC) no siempre da buenos resultados, necesitando con frecuencia múltiples sesiones y tratamientos complementarios como la nefrolitotomía percutánea (NLP) y disolución mediante irrigación. En general se acepta que en cálculos piélicos únicos y en adultos, la LEOC estaría indicada para tamaños inferiores a 1,5 cm. La prevalencia de aparición de nuevos cálculos se sitúa entre 0,42 episodios al año en los hombres y 0,21 en las mujeres, y la mayoría de los pacientes sufrirá una enfermedad calculosa recidivante durante toda su vida ${ }^{22}$. Debido precisamente a la alta tasa de recidiva en estos pacientes es más aconsejable adoptar medidas poco invasivas para no hipotecar la vía excretora y la función renal, en la medida de lo posible. Esta terapia conservadora estaría basada en una alta ingesta de líquidos, alcalinización de la orina, y en la utilización de fármacos con acción quelante, medidas todas ellas encaminadas a disminuir los niveles de cistina libre en la orina con la consiguiente disminución de 
la recurrencia y la morbilidad de la enfermedad.

Terapia conservadora: a) Dieta y líquidos; b) Alcalinización de la orina; y c) Compuestos quelantes.

a) Dieta y líquidos. Independientemente del tipo de cálculo que presente un paciente el aumento de la ingesta de líquidos es la primera medida a instaurar, con la consiguiente disminución de la sobresaturación de cualquier sal urinaria ${ }^{23}$. En los pacientes con cistinuria se recomienda un volumen de líquido diario de entre 3-3,5 litros, superior a los 2 litros/día recomendados en cualquier otra litiasis ${ }^{24}$. La dieta del paciente ha de ser equilibrada, ajustándola a los requerimientos nutricionales básicos dependiendo de la talla, edad, sexo y constitución del paciente. En estos pacientes se ha sugerido el uso de una dieta restrictiva en proteínas y en aminoácidos, en concreto baja en metionina (por ser la precursora biosintética de la cisteína) y en cisteína ${ }^{25}$. Sin embargo, hasta la fecha, no se ha documentado ninguna evidencia de que esta dieta restrictiva suponga una mejoría apreciable en el curso clínico de la enfermedad.

b) Alcalinización de la orina. El objetivo que se persigue con la alcalinización es mantener un $\mathrm{pH}$ urinario entre 7 y 7,5 y sin que supere el valor de 8 . Ya que a pesar de la elevada solubilidad de la cistina a $\mathrm{pH}=8$ (de hasta $1.000 \mathrm{mg} / \mathrm{L}$ ), el paciente presentaría un riesgo elevado de precipitación de sales de calcio y fosfato con la consiguiente formación de cálculos de estas sales y lo que a su vez aumentaría el riesgo de infección. Es recomendable igualmente utilizar sales de potasio como alcalinizadoras en lugar de utilizar sales de sodio. Ello es debido a que el incremento en la ingesta de sodio conlleva un aumento en la excreción urinaria de cistina y aumento de la formación de cálculos, siendo por tanto recomendable que la dieta tenga un bajo contenido de sodio $^{26,27}$.

Como agentes alcalinizadores se están empleando en la actualidad el citrato de potasio y la acetazolamida. El citrato de potasio desde su aprobación por la F.D.A en 1995 sólo o en combinación con otros fármacos está siendo ampliamente utiliza- $\mathrm{do}^{28}$. Se comienza con una dosis inicial de $60-80 \mathrm{mEq} /$ día dividida en 4 dosis y/o una dieta rica en zumos cítricos ${ }^{29}$. La acetazolamida (Diamox, Lederle Laboratories, San Francisco, California, USA) es un inhibidor de la anhidrasa carbónica con efecto diurético suave y alcalinizante. Por su efecto hipercalciúrico no es de uso común en las litiasis, pero sí está indicado su uso en el tratamiento alcalinizante de la cistinuria asociado a citrato potásico, a dosis de 250 mg antes de acostarse $\mathrm{e}^{30,31}$.

c) Compuestos quelantes. Son moléculas derivadas del tiol que interaccionan con la cistina reduciéndola y formando con la cisteína (Cys) resultante un puente disulfuro. De esta forma disminuyen la concentración de cistina libre, y forman compuestos derivados más solubles que son fácilmente eliminados por la orina. Los fármacos principales que se están empleando con este fin son la D-penicilamina, tiopronina ( $\alpha$-mercaptopropionilglicina, $\alpha-\mathrm{MPG}$ ), bucilamina, y el captopril ${ }^{32,33}$.

La penicilamina es un producto de degradación del antibiótico penicilina. Es un fármaco sin actividad antimicrobiana, el cual presenta acciones de tipo quelante y anti-inflamatorio en algunas formas de artritis (artritis refractarias) ${ }^{34}$. Estructuralmente se relaciona con la Cys, por lo que se la conoce también como $B$, ß-dimetilcisteína (Figura 2B). Se usa principalmente en el tratamiento de la artritis reumatoide juvenil, y en la enfermedad de Wilson ${ }^{35}$, además del uso que presenta en los pacientes con cistinuria. La penicilamina forma compuestos con la Cys resultante que son 50 veces más solubles que la cistina ${ }^{36,37}$. Las dosis de penicilamina empleadas en el tratamiento de la cistinuria son de $2 \mathrm{~g} /$ día en adultos, oscilando entre 1 y $4 \mathrm{~g} /$ día. En los enfermos pediátricos la dosis media es de $30 \mathrm{mg} /$ $\mathrm{kg} /$ día. En ambos casos, la dosis diaria se debe administrar en 4 tomas y si ello no es posible, en tres, administrando una dosis mayor por la noche. Si se necesita reducir la dosis debido a las reacciones adversas, se procurará mantener la dosis de la noche. Las dosis se deben individualizar de forma que la excreción diaria de cistina sea de 100-200 mg en los pacientes sin historia 
de cálculos renales y de $100 \mathrm{mg}$ en los que hayan tenido cálculos y/o dolores renales. Es conveniente iniciar el tratamiento con una dosis de $250 \mathrm{mg} /$ día e ir aumentando progresivamente la dosis hasta obtener un control adecuado de la cistinuria ${ }^{38}$. De esta forma se consigue un mejor control sobre los efectos del fármaco y se reducen las reacciones secundarias. Los pacientes con cistinuria deben beber copiosamente en particular antes de acostarse ya que la orina nocturna es más concentrada que la diurna. Además, cuanto mayor sea la ingesta de líquidos, menores serán las dosis de penicilamina necesarias. Se han descrito reacciones adversas en el $50 \%$ de los $\operatorname{casos}^{37,39}$. Entre los efectos secundarios descritos se encuentran: fiebre, hematuria, leucopenia, trombocitopenia, anemia hemolítica, hepatitis, y pancreatitis entre otros $^{14}$. Su empleo prolongado resulta en una deficiencia de vitamina B6, así que se recomienda un suplemento con esta vitamina de unos $50 \mathrm{mg} /$ día $^{7}$.

La $\alpha$-mercaptopropionilglicina representa la segunda generación de agentes quelantes; se comercializa con el nombre de Thiola en EEUU y tiopronina en Europa (se expende en cápsulas de $250 \mathrm{mg}$ ). Presenta unas características químicas similares a la penicilamina, y un mecanismo de acción similar pero con una menor toxicidad. La dosis inicial que se recomienda es de $250 \mathrm{mg} /$ día, y un incremento gradual hasta alcanzar la dosis de 800-1.200 mg/ día ${ }^{26}$. Parece ser que el efecto terapéutico quelante de la tiopronina, al menos en un modelo de ratón se ve reforzado si se combina conjuntamente con agentes alcalinizantes ${ }^{40}$. Este hecho refuerza la idea de utilizar conjuntamente los compuestos alcalinizantes con los quelantes para así potenciar el efecto de estos últimos.

La bucilamina representa la tercera generación de agentes quelantes con una acción superior a la penicilamina y tiopronina ${ }^{41,42}$, pero sin embargo ha tenido hasta la fecha un uso muy limitado y la información disponible sobre su empleo es muy pequeña.

El captopril además de su función hipotensora tiene una función quelante, al unir- se a la cistina y formar compuestos con ella 200 veces más hidrosolubles ${ }^{28}$. Por lo que su uso está indicado en los pacientes cistinúricos que presentan además hipertensión teniendo un doble efecto.

Dentro de los agentes quelantes usados para el tratamiento de pacientes cistinúricos la tiopronina es hasta la fecha la que ha presentado mejores resultados clínicos $y$ con menores efectos secundarios ${ }^{43}$. Sin embargo se han obtenido muy buenos resultados y con una tasa de efectos adversos muy pequeña, en un estudio llevado a cabo en un grupo de niños cistinúricos empleando un tratamiento conservador con penicilamina a dosis crecientes ${ }^{44}$. En cuanto a los resultados obtenidos con el empleo del captopril con efecto quelante los resultados son controvertidos. Así Cohen $\mathrm{y} \mathrm{col}^{45}$ obtienen muy buenos resultados en pacientes adultos e hipertensos, y sin embargo Coulthard y col $^{46}$ obtienen descensos muy pequeños en los niveles de cistina en pacientes pediátricos no hipertensos tratados con captopril. En otros estudios con niños cistinúricos tratados con captopril y terapia conservadora sin embargo se obtienen muy buenos resultados con una baja recidiva en la formación de nuevos cálcu$\operatorname{los}^{47,48}$. Por lo tanto estos datos tan contradictorios pueden indicar que el captopril puede tener efecto o no en la disminución de los niveles de cistina independientemente de su efecto hipotensor por causas todavía no conocidas, siendo necesarios estudios más exhaustivos de su uso.

\section{CONSIDERACIONES FINALES}

La cistinuria se encuentra entre los errores congénitos del metabolismo de los aminoácidos más frecuentes, siendo su única manifestación clínica conocida la formación de cálculos de cistina. Ante un paciente con un diagnóstico de cistinuria no se pude utilizar el mismo planteamiento terapéutico que ante cualquier otro tipo de litiasis renal, ya que son cálculos de extrema dureza y su alta tasa de recidiva no permiten emplear técnicas convencionales de litotricia. Por ello es muy importante, que una vez hecho un diagnóstico acertado se pongan en mar- 
cha las medidas de prevención para minimizar su recurrencia: alta ingesta de líquidos, alcalinización de la orina, y uso de agentes quelantes. Habría que individualizar las medidas terapéuticas, siendo de especial relevancia la elección del quelante adecuado. Ya que si bien han demostrado suficiente eficacia, no son igualmente tolerados por todos los pacientes con presentación de diferentes reacciones adversas, las cuales habría que minimizar ajustando la dosis o bien cambiando de agente quelante. Es muy importante igualmente para poder emplear conjuntamente estas tres medidas contar con la colaboración del paciente. Sin embargo, en la edad pediátrica donde se diagnostican la mayoría de los casos, mantener una elevada ingesta de líquidos es muy difícil de llevar a la práctica. Por lo que la alcalinización y el uso de compuestos quelantes sería la alternativa de tratamiento. Igualmente es necesario llevar un control periódico de los niveles de cistina en orina para ajustar las dosis del tratamiento y evaluar la respuesta al mismo.

\section{BIBLIOGRAFÍA}

1. SEgal ST. Cystinuria. En: Scriver $\mathrm{CH}$, Beaudet AL, Sly WS, Valle D, editores. The metabolic and molecular bases of inherited diseases New York: McGraw-Hill, 1995: 3581-3601.

2. Rousaud F, Palacin M, Nunes V. Cistinuria. Nefrología 2003; 23 Supl.1: 52-59.

3. GoOdyer P. The molecular basis of cystinurie. Nephron Exp Nephrol 2004; 98: 45-49.

4. SAYER JA. The genetics of nephrolithiasis. Nephron Exp Nephrol 2008; 110: 37-43.

5. Matoo A, Goldfarb DS. Cystinuria. Semin Nephrol 2008; 28: 181-191.

6. BRÖER S. Amino acid transport across mammalian intestinal and renal epithelia. Physiol Rev 2008; 88: 249-286.

7. Orts Costa JA, ZúÑiga Cabrera A, Martínez de la Cámara y Salmerón J. Actualización de la cistinuria: aspectos clínicos, bioquímicos y genéticos. An Med Interna (Madrid) 2003; 20: 317-326.

8. Fernández E, Carrascal M, Rousaud F, Abián J, Zorzano A, PALAcin M et al. rBAT- $\mathrm{b}^{0,+}$ AT heterodimer is the main apical reabsorption system for cystine in the kidney. Am J Physiol Renal Physiol 2002; 283: F540-F548.
9. Vicente Calderón C. Tubulopatías. Curso precongreso. XXX Congreso Nacional de Nefrología Pediátrica (1). Bol Pediatr 2010; 50: 62-65.

10. Rodríguez Fernández LM, LAPEÑa LóPEZ dE ARMENTIA S. Cistinuria. Revisión teórica. Bol Pediatr 1997; 37: 73-77.

11. Assimos DG, Leslie SW, Ng C, Streem SB, Hart LJ. The impact of cystinuria on renal function. $\mathrm{J}$ Urol 2002; 168: 27-30.

12. PuRohit RS, Stoller ML. Laterality of symptomatic cystine calculi. Urology 2003; 62: 421-424.

13. Dello Strologo L, Pras E, Pontesilli C, Beccia E, Ricci-Barbini V, De SAnctis L et al. Comparison between SLC3A1 and SLC7A9 cystinuria patients and carriers: a need for a new classification. J Am Soc Nephrol 2002; 13: 2547-2553.

14. Shekarriz B, Stoller ML. Cystinuria and other noncalcareous calculi. Endocrinol Metab Clin North Am 2002; 31: 951-977.

15. Chillarón J, Font-Llitjós M, Fort J, Zorzano A, GoldFarb DS, Nunes V et al. Pathophysiology and treatment of cystinuria. Nat Rev Nephrol 2010; 6: 424-434.

16. Palacín M, Goodyer P, Nunes V, Gasparini P. Cystinuria. En: Scriver CR, Beaudet AL, Sly SW, Valle D, Childs B, Kinzler KW, Vogelstein B, editores. The Molecular and Metabolic Bases of Inherited Disease, $8^{\text {th }}$ edition. New York: McGraw-Hill, 2001: 4909-4932.

17. Guillén M, Corella D, Cabello ML, García Am, HERNÁNDEZ-YAGo J. Reference values of urinary excretion of cystine and dibasic aminoacids: classification of patients with cystinuria in Valencian Community, Spain. Clin Biochem 1999; 32: 25-30.

18. Finocchiaro R, D'eufemia P, Celli M, Zaccagnini M, Viozzi L, Troiani P et al. Usefulness of cyanidenitroprusside test in detecting incomplete recessive heterozygotes for cystinuria: a standardized dilution procedure. Urol Res 1998; 26: 401-405.

19. Francés F, Corella D, Carrasco P, Sáiz C, Guillén M. Modulación de la expresión fenotípica del paciente con cistinuria: influencia de la intervención terapéutica y de la dieta. Arch Esp Urol 2007; 60: 109-118.

20. Martínez-Llamas MS, Cabrera Morales CM, Bravo Soto JA, Pedrinaci S. Cystinosis: diagnosis through the measurement of the leukocyte cystine content by HPLC. Med Clin (Barc) 2004; 123: 97-99.

21. Cochat P, Pichault V, Bacchetta J, Dubourg L, SAвот $\mathrm{JF}, \mathrm{S}_{A B A N} \mathrm{C}$ et al. Nephrolithiasis related to inborn metabolic diseases. Pediatr Nephrol 2010; 25: 415-424. 
22. Dello Strologo L. Cystinuria. Orphanet Encyclopedia. September 2003: http://www: orpha.net/data/patho/GB/uk-cystinuria.pdf.

23. EvAN AP. Physiopathology and etiology of stone formation in the kidney and the urinary tract. Pediatr Nephrol 2010; 25: 831-841.

24. García JL, Vázquez Martul M, Baeza J, García P. Litiasis renal coraliforme en cistinuira. Eficacia del tratamiento conservador. Nefrología 1994; 14: 224-227.

25. Rodman JS, Blackburn P, Williams JJ, Brown A, PospischIL MA, PETERSON CM. The effect of dietary protein on cystine excretion in patients with cystinuria. Clin Nephrol 1984; 22: 273-278.

26. Ng CS, StreEm SB. Medical and surgical therapy of the cystine stone patient. Curr Opin Urol 2001; 11: 353-358.

27. Lindell A, Denneberg T, Edholm E, Jeppsson JO. The effect of sodium intake on cystinuria with and without tiopronin treatment. Nephron 1995; 71: 407-415.

28. Ibarz Servio L, Conte Visús A, Arzoz Fabregas M, Ruiz Marcellán FJ. Tratamiento médico de la litiasis renal. Annals d'Urologia 2006; 6: 1-16.

29. Fuellstedt E, Denneberg T, Jeppsson JO, Tiselius HG. A comparison of the effects of potassium citrate and sodium bicarbonate in the alkalinization of urine in homozygous cystinuria. Urol Res 2001; 29: 295-302.

30. Arrabal M. Tratamiento médico de la litiasis urinaria. En: Astobieta A, Rodríguez JM, Resel L, editores. Tratado de Farmacoterapia en Urología. Madrid: Luzón 5 Ediciones SA, 2001: 753-798.

31. Sterret SP, Penniston KL, Wolf Js JR, NaKada SY. Acetazolamide is an effective adjunct for urinary alkalization in patients with uric and cystine stone formation recalcitrant to potassium citrate. Urology 2008; 72: 278-281.

32. Rogers A, Kalakish S, Desai RA, Assimos DG. Management of cystinuria. Urol Clin North Am 2007; 34: 347-362.

33. Biyani CS, Cartledge JJ. Cystinuria: diagnosis and management. EAU-EBU Update Series 2006; 4: 175-183.

34. Merino R, García-Consuegra J. Actualización del tratamiento farmacológico de las artritis crónicas infantiles. An Esp Pediatr 1999; 50: 1-3.

35. Pérez-Aguilar F. Enfermedad de Wilson: consideraciones fisiopatológicas, clínicas y terapéuticas. Gastroenterol Hepatol 2003; 26: $42-51$.
36. Dello Strologo L, Laurenzi C, Legato A, Pastore A. Cystinuria in children and young adults: success of monitoring free-cystine urine levels. Pediatr Nephrol 2007; 22: 1869-1873.

37. Joly D, Rieu P, MéJean A, Gagnadoux MF, Daudon M, Jungers P. Treatment of cystinuria. Pediatr Nephrol 1999; 13: 945-950.

38. REYNolds TM. Chemical pathology clinical investigation and management of nephrolithiasis. J Clin Pathol 2005; 58: 134-140.

39. Zelikovic I. Aminoaciduria and glycosuria. En: Anver ED, Harmon WE, Niaudet P, editores. Pediatric nephrology, $5^{\text {th }}$ ed. Philadelphia, Lippincott Williams and Wilkins, 2004: 701729.

40. Wendt-Nordahl G, Meister L, Michel S, Knoll T. Evaluation of alkalinization and chelating agent therapy for cystinuria in animal model (abstract n²340). Eur Urol 2009; S8: 205.

41. Sakhaee K, Sutton RAL. Pathogenesis and management of cystinuria. En: Coe FL, Favus MJ, Pak CYC, Parks JH, Preminger GM, editores. Kidney stones: medical and surgical management. Philadelphia, Pennsylvania: LippincottRaven Publishers, 1996: 1007-1017.

42. Koide T, Yamaguchi S, Utsonomiya M, Yoshioka T. A new therapeutic agent for cystinuria. En: Urolithiasis 2. Proceedings of the VIIth International Symposium on Urolithiasis; 2427 August 1992; Cairns, Australia. New York, USA: Plenum Press, 1994: 571-574.

43. Tiselius HG. New horizons in the management of patients with cystinuria. Curr Opin Urol 2010; 20: 169-173.

44. Deberardinis RJ, Coughuin CR 2Nd, Kaplan P. Penicillamine therapy for pediatric cystinuria: experience from a cohort of American children. J Urol 2008; 180: 2620-2623.

45. Cohen TD, Streem SB, Hall P. Clinical effect of captopril on the formation and growth of cystine calculi. J Urol 1995; 154: 164-166.

46. Coulthard MG, Richardson J, Fleetwood A. The treatment of cystinuria with captopril. Am J kidney Dis 1995; 25: 661-662.

47. Sanchez C, Ruiz R, Gutierrez A, Martínez C, Toro V, Perez G. Cystine calculi. Prevention with captopril. Clinical case. Actas Urol Esp 2000; 24: 190-196.

48. Printza N, Koukourgianni F, Papathanasiou A, Augoustides-Savvopoulou P, Papachristou F. Efficacy of captopril therapy in cystinuria lithiasis. A case report. Hippokratia 2007; 11: 83-85. 